\title{
Protée
}

\section{Jean-Pierre Séguin : Portraits-émergents sur détails-objets}

\section{Michaël La Chance}

Volume 32, numéro 2, automne 2004

L’archivage numérique : conditions, enjeux, effets

URI : https://id.erudit.org/iderudit/011172ar

DOI : https://doi.org/10.7202/011172ar

Aller au sommaire du numéro

Éditeur(s)

Département des arts et lettres - Université du Québec à Chicoutimi

ISSN

0300-3523 (imprimé)

1708-2307 (numérique)

Découvrir la revue

Citer cet article

La Chance, M. (2004). Jean-Pierre Séguin : Portraits-émergents sur détails-objets. Protée, 32(2), 47-58. https://doi.org/10.7202/011172ar d'utilisation que vous pouvez consulter en ligne.

https://apropos.erudit.org/fr/usagers/politique-dutilisation/ 


\title{
JEAN-PIERRE SÉGUIN
}

\section{Portraits-émergents sur détails-objets}

\author{
une présentation de Michaël La Chance
}


Il fut un temps où la technique était au service de la peinture, cette dernière visait à produire une expérience de contemplation. La perfection technique permettait d'éviter que le détail ne vienne provoquer une attention excessive au procédé. Selon cette conception encore très répandue, l'unité de l'œuvre relève d'une expérience spirituelle autant qu'esthétique, que ne peut expliquer une accumulation de détails. Contre cet idéalisme dans la peinture, Jean-Pierre Seguin place au premier plan le détail, qu'il veut sériel, objectal et commun - afin que le spectateur se rappelle qu'il constitue ses figures -, non pas à partir de rien, mais à partir du monde d'objets et d'usages que nous appelons quotidien.

Le fait même de figurer apparaît finalement très abstrait, lorsqu'il est contrasté avec la dimension très concrète des éléments constitutifs de la figure. Depuis plusieurs années, Jean-Pierre Séguin est fasciné par le rôle du support dans le portrait, quand le support peut déconstruire la figuration proposée ou la recontextualiser de façon totalement inattendue. Les grandes choses sont composées de petits détails, ainsi - semble-t-il - l'identité humaine est composée de mille composantes rassemblées qui permettent de croire à une essence de l'humain. Si le pixel est la plus petite unité perceptible à l'écran... ainsi Séguin s'intéresse à faire varier ce qu'on pourrait appeler le «pictel », c'est-à-dire la plus petite unité picturale qui vient constituer le tableau: que cela soit un code barres, un bouton, une pièce de puzzle... Alors l'image repose sur des objets. Inversement, les objets sont davantage que les accessoires nécessaires à la mise en scène de notre monde, ils figurent déjà ce monde - ce sont les inducteurs anthropomorphiques d'une certaine invention de l'humain.

Cette démarche semble motivée par le débat actuel en science autour de la notion d'émergence: ou bien nous devons admettre que les organismes, phénomènes, événements (y compris nos événements psychiques) seraient le résultat d’une convergence d'effets produits par une multiplicité d'automates, ou bien nous devons admettre plutôt - comme dans ce cas-ci - que la figure est un phénomène émergent. Cette démarche semble également motivée par l'apparition de la photographie numériqueà une époque où nous avons développé un fétichisme du pixel. Quelle différence entre une image 300ppp (pixels par pouce) et une image au pastel? La différence semble surtout de résolution. Et puis, comment en sommes-nous venus à tolérer des images à 100ppp: nous aurions besoin de rendre les images plus palpables pour ne pas succomber à l'illusion? Aujourd'hui les images sont plus nombreuses et commandent une lecture plus rapprochée, quand nous sommes littéralement invités à rentrer dans l'image - ce que nous propose la réalité virtuelle. Dans l'œuvre quelque peu ironique de Séguin, cette lecture rapprochée nous fait découvrir un tableau plus abstrait qu'il ne le semblait et tout à la fois plus concret.

Nombre de ces figures réalisées avec des objets sont des portrait d'artistes. Ainsi, à ce processus, Séguin convie l'histoire de l'art en la personne d'artistes contemporains: il écrit l'histoire au présent, sans que l'on puisse dire que ce soit une histoire monumentale, puisque les «monuments», quelque peu humoristiques, apparaissent ici faits de boutons. On pourrait dire alors que la représentation est déconstruite, réduite à des séries, des dispersions, des proliférations... bref, qu'elle est déboutonnée.

Peut-être que tout est figure lorsqu'on se tient à une certaine distance des choses, et que tout redevient objet lorsqu'on réduit cette distance. C'est une question de distance et aussi d'assignation. On s'étonne de la capacité de figurer des pièces assemblées, il y a un détournement de l'objet en image qui rend perplexe. Une fausse familiarité s'installe d'emblée: la culture des objets est partagée de tous, elle induit un ensemble prédéfini de réflexes culturels et perceptifs. Séguin joue sur cette ambiguïté de l'image, quand celle-ci se révèle supportée par de multiples systèmes de codification, et tout à la fois propose des tableaux saisissants, qui produisent les effets de présence que l'on peut attendre de la figure. Est-ce un dépuzzlage de la représentation?

M. L. 


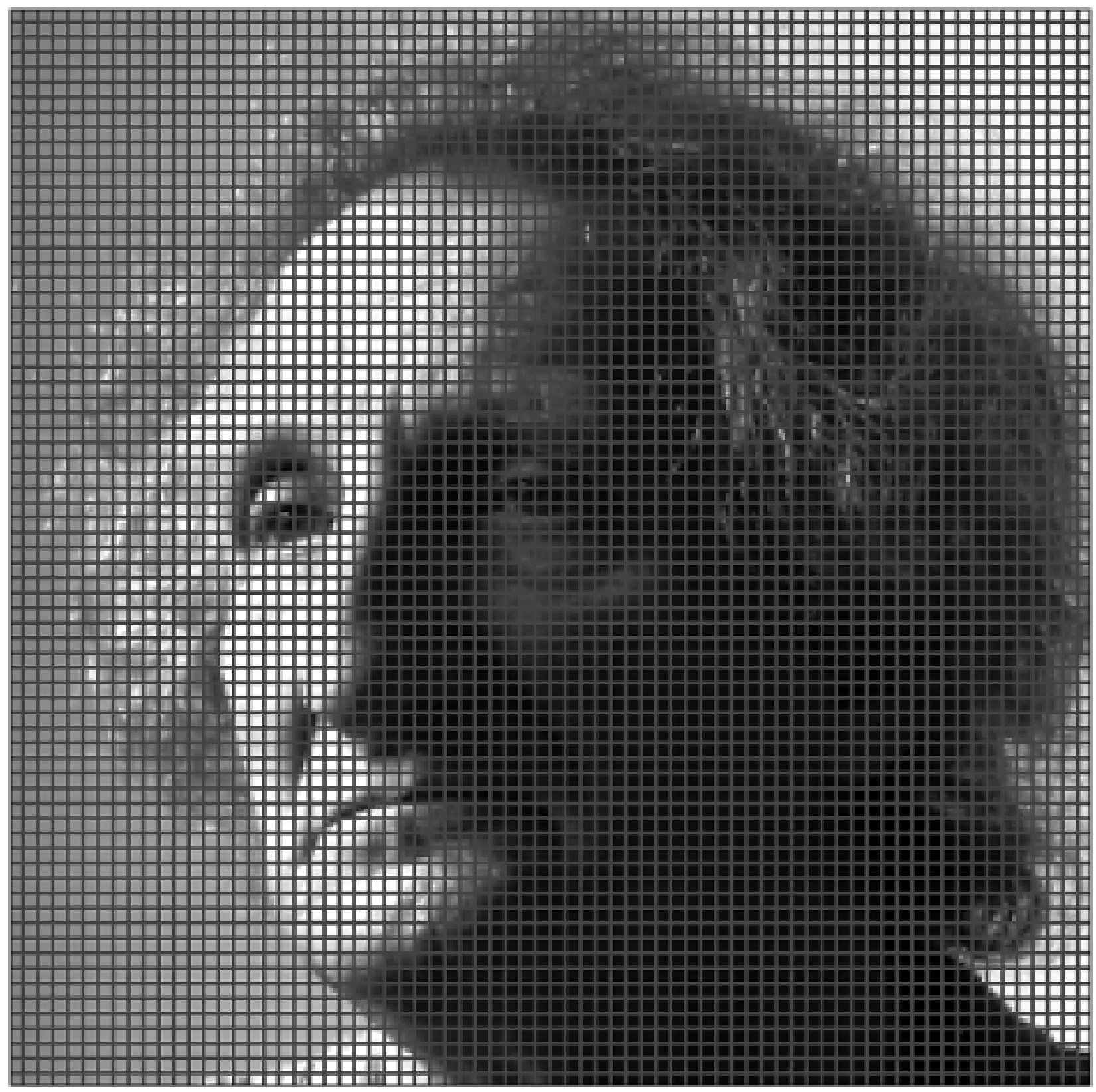

GM, 2003, encre archive sur toile, 122 × 122cm (détail de l'œil gauche en page couverture). 


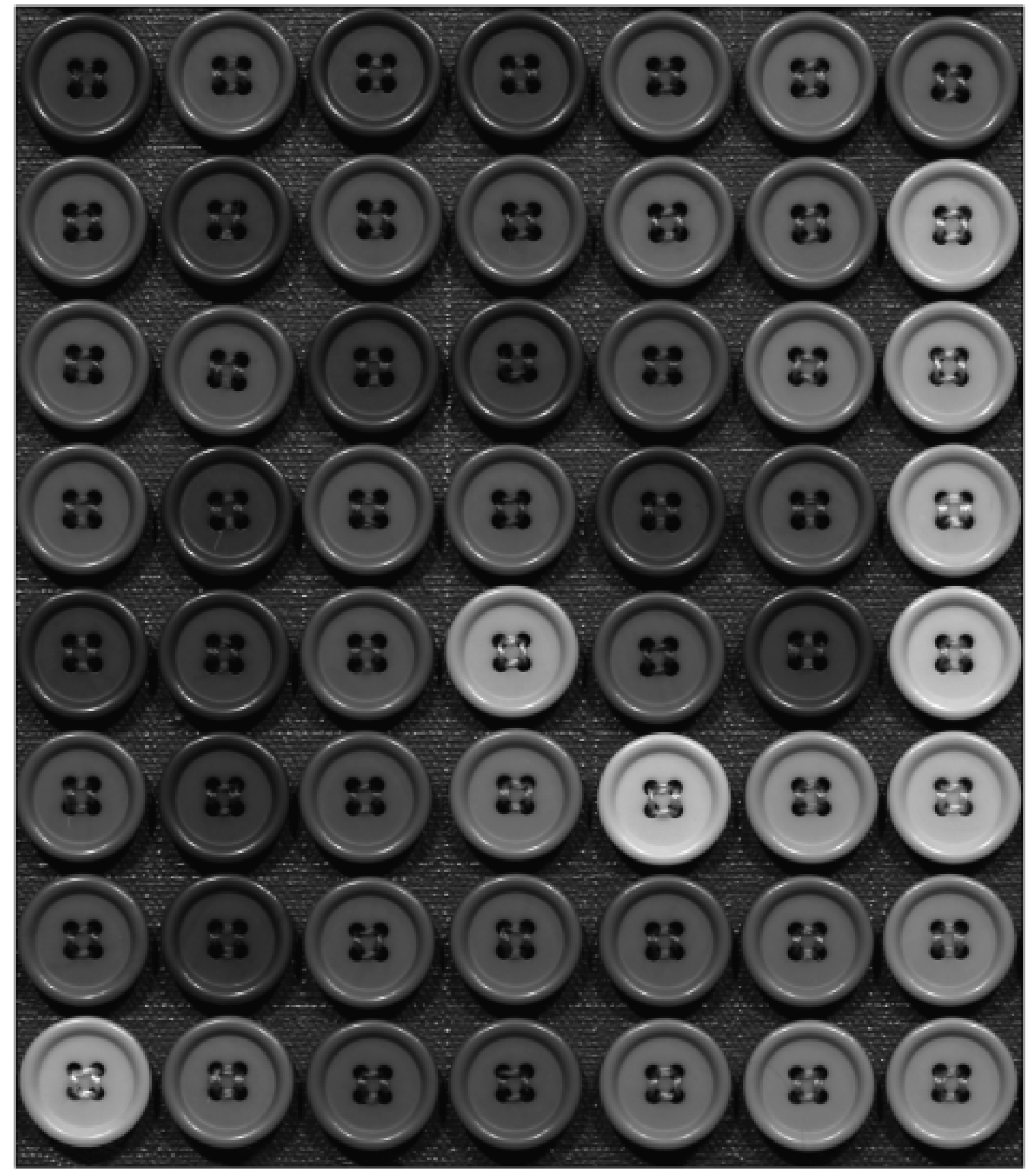

MC (détail) 2002-2003, boutons sur toile, 130 x 168cm. 


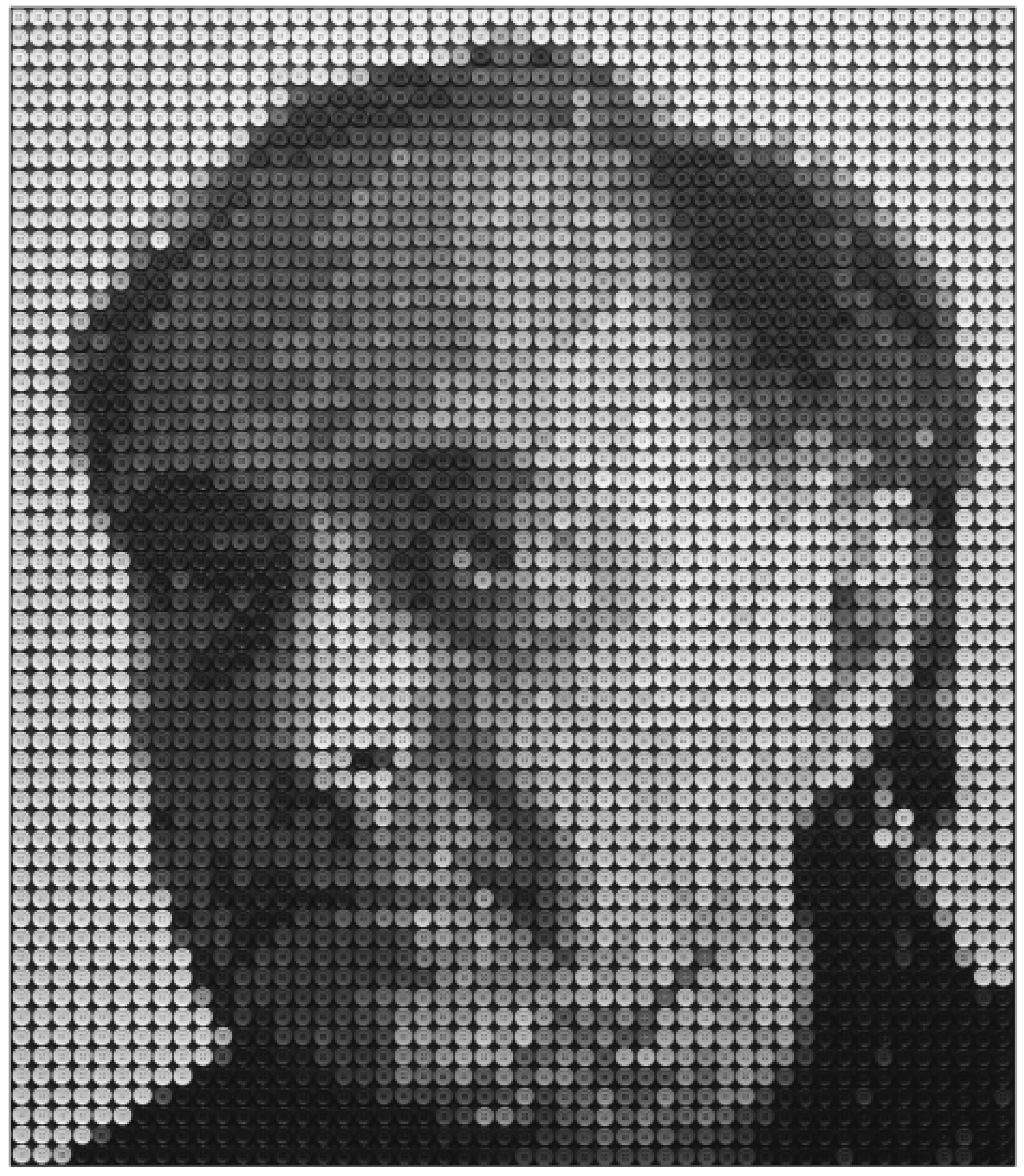

MC, 2002-2003, boutons sur toile, 130 x 168cm (commandite de Boutons du Canada Itée). 


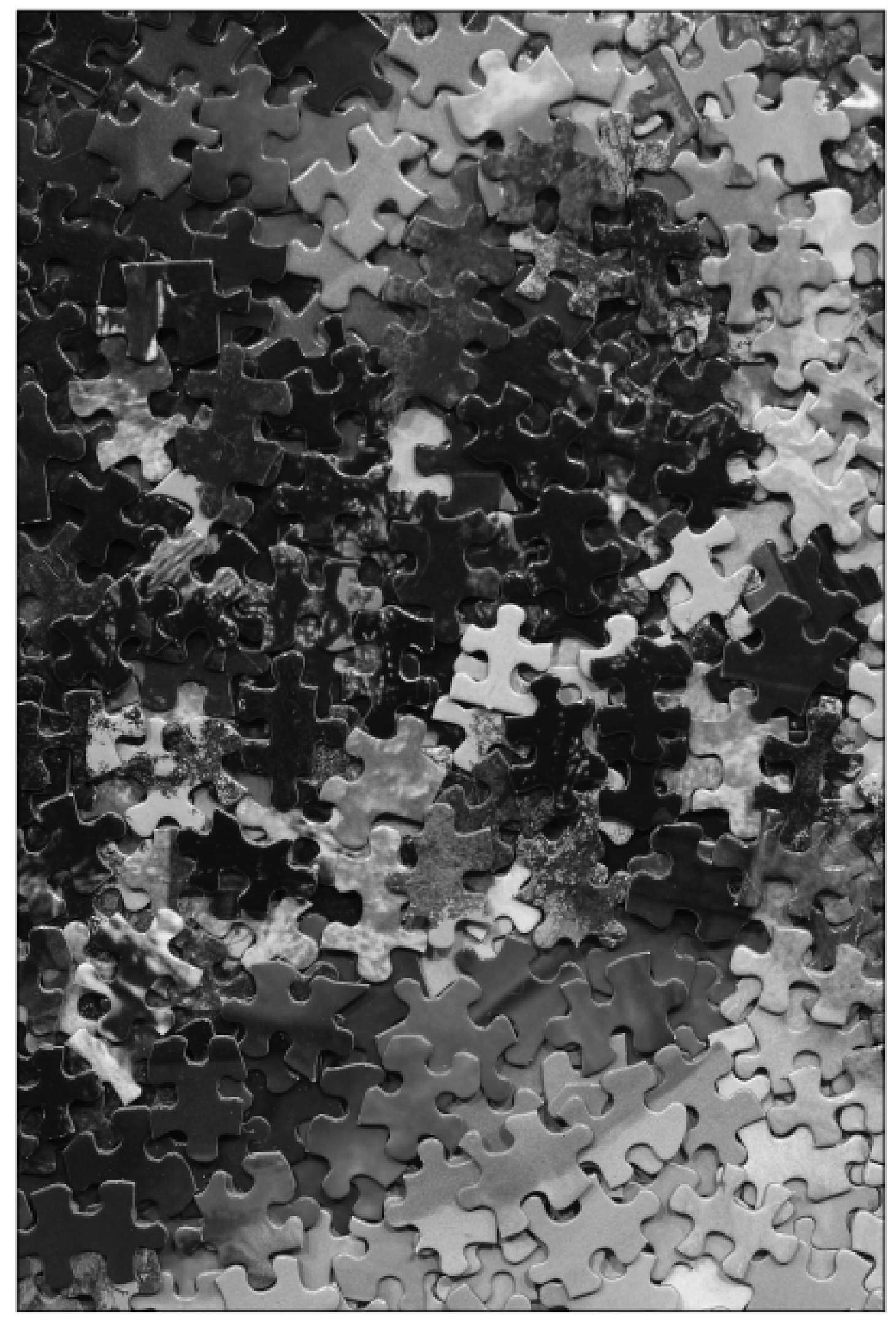

Portrait nabis de MG (détail), 2001, collage de pièces de casse-tête sur bois, 76 x 112cm. 


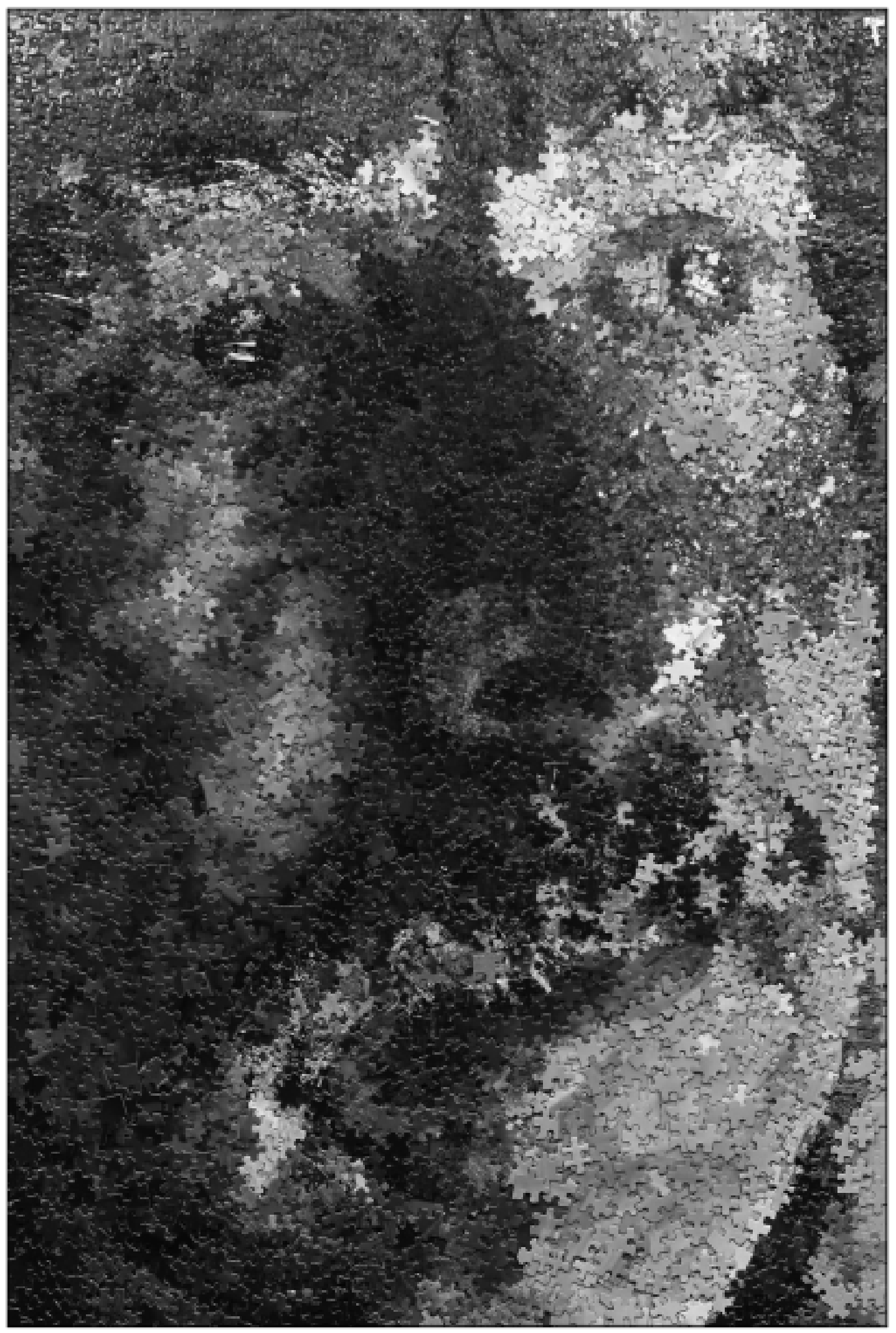

Portrait nabis de MG, 2001, collage de pièces de casse-tête sur bois, 76 x 112cm. 


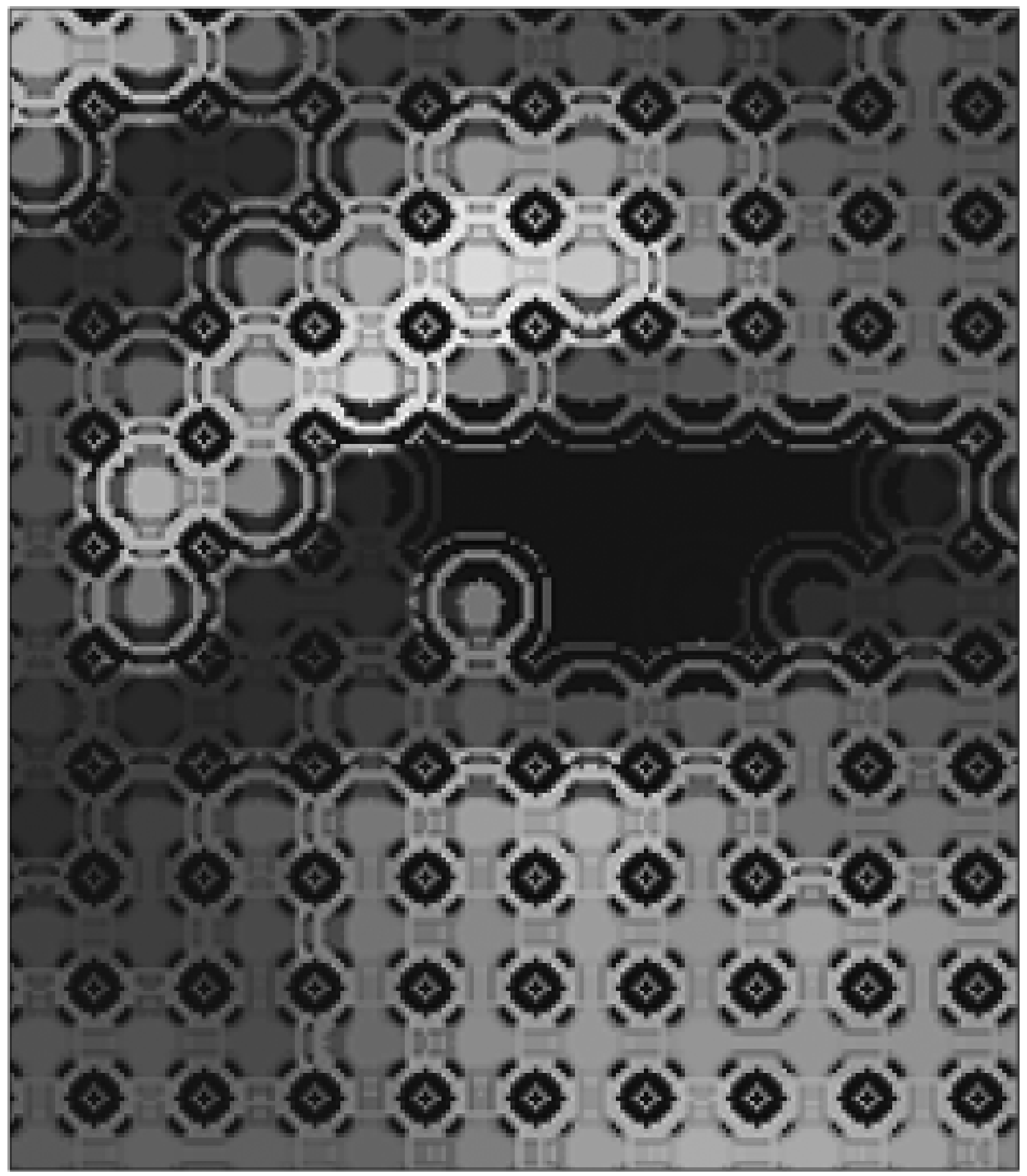

$H T$ (détail), 2002, encre archive sur toile, $137 \times 171 \mathrm{~cm}$ 


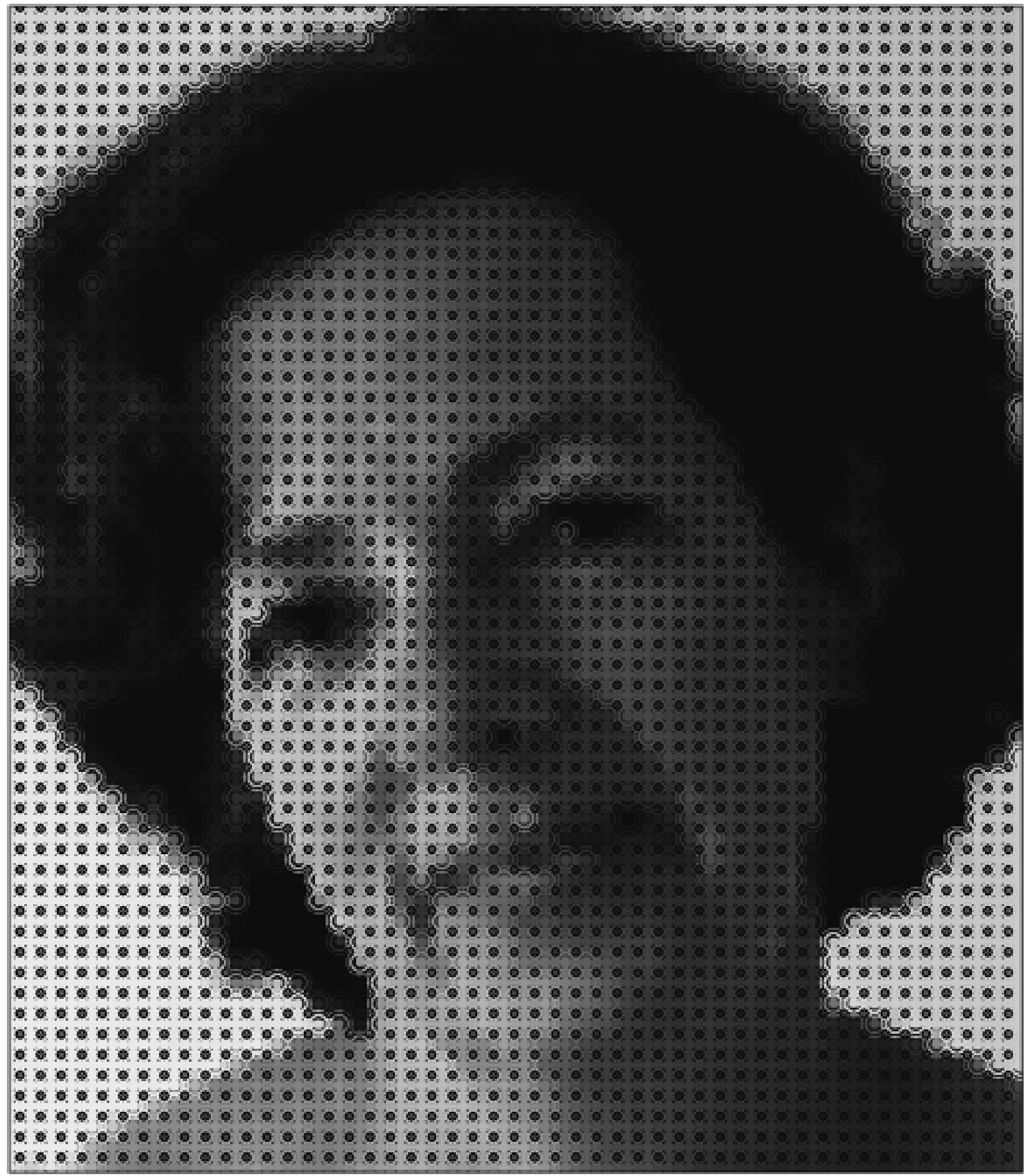

$H T, 2002$, encre archive sur toile, $137 \times 171 \mathrm{~cm}$. 


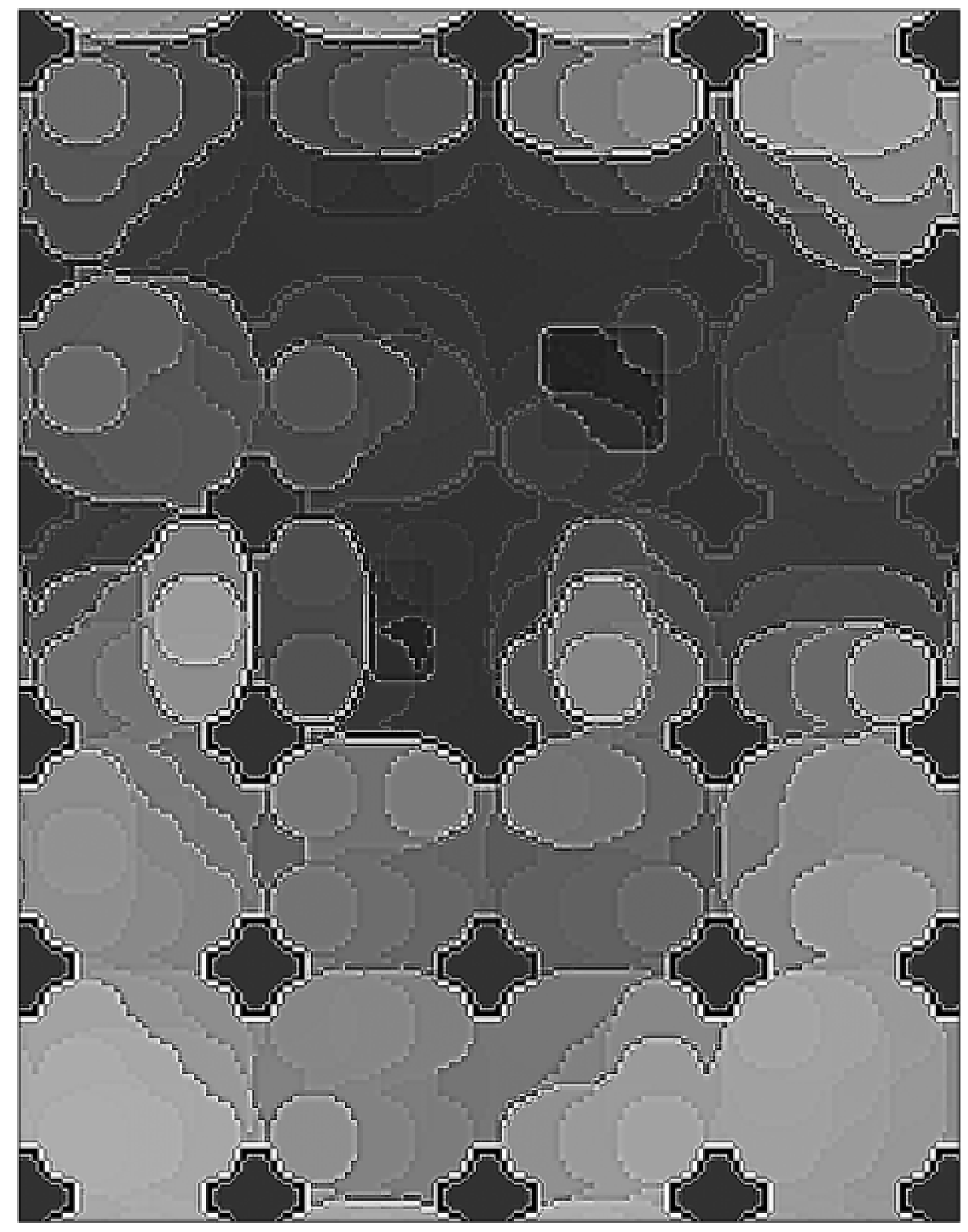

CS (détail), 2002, encre archive sur toile, $142 \times 183 \mathrm{~cm}$ 


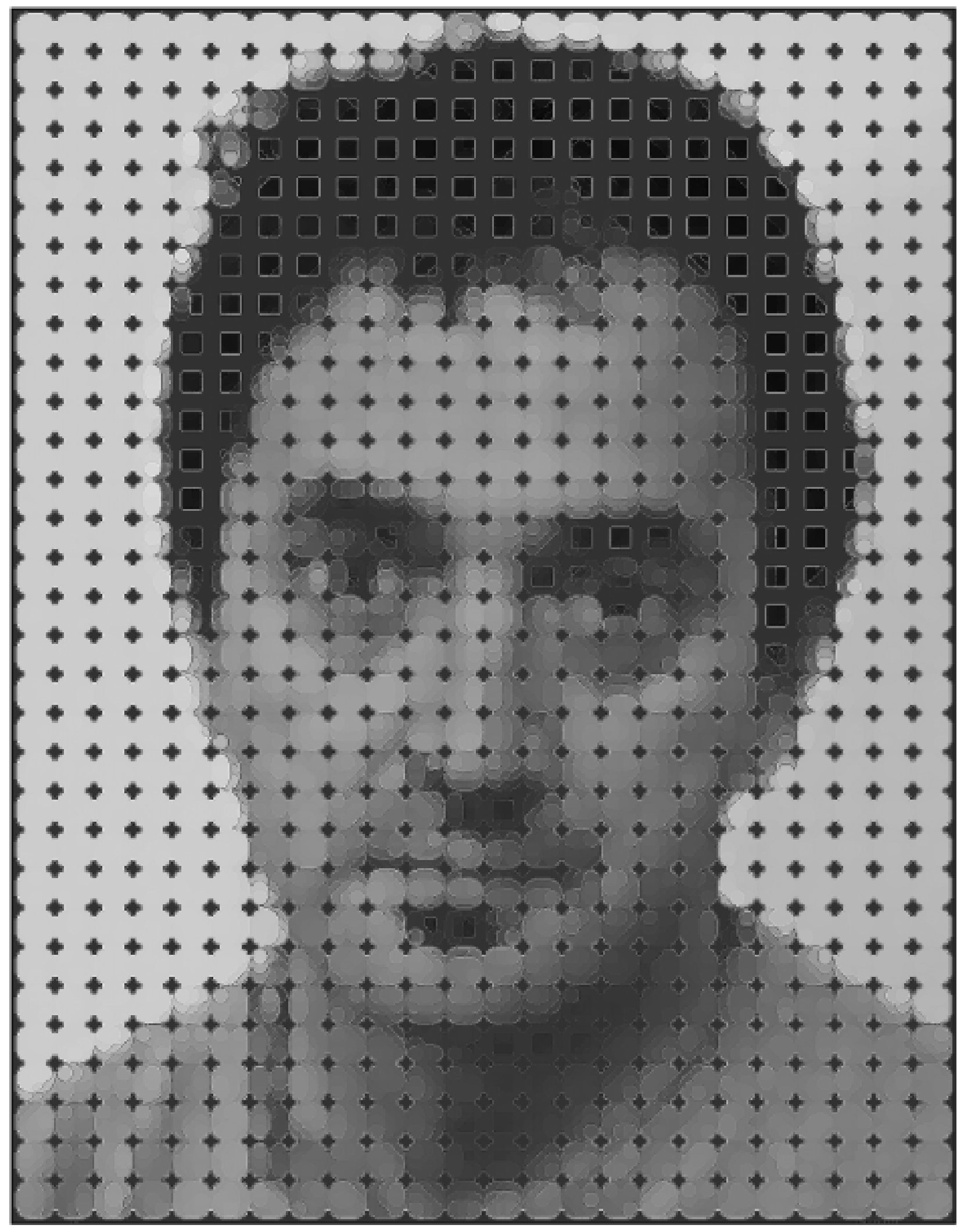




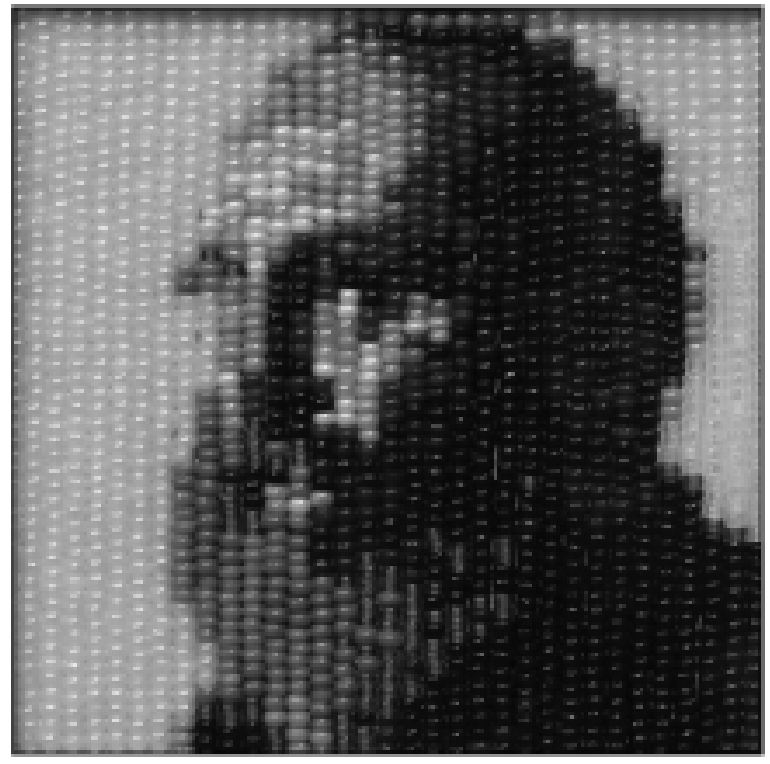

Étude pour E, 2003, perles de couleur, $7 \times 7 \mathrm{~cm}$.

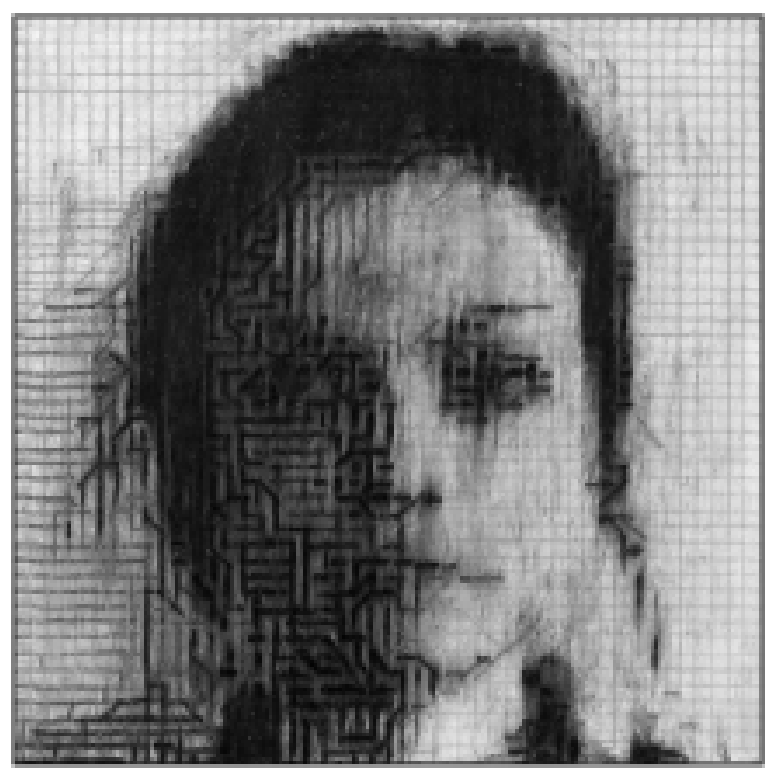

$C D$ étude graphite, 2004, crayon sur papier, $8 \times 8 \mathrm{~cm}$.

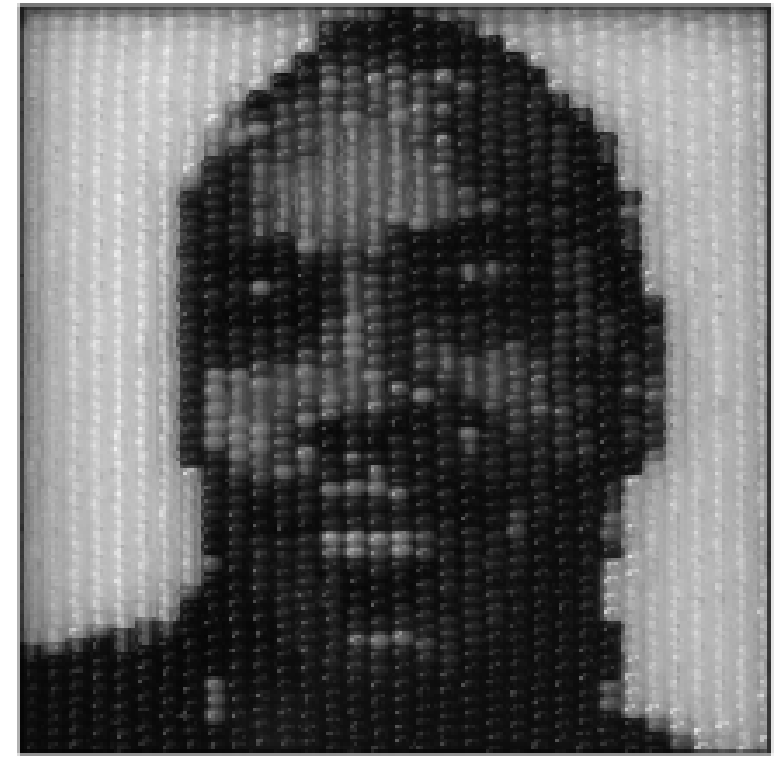

Étude pour DF, 2003, perles de couleur, $7 \times 7 \mathrm{~cm}$.

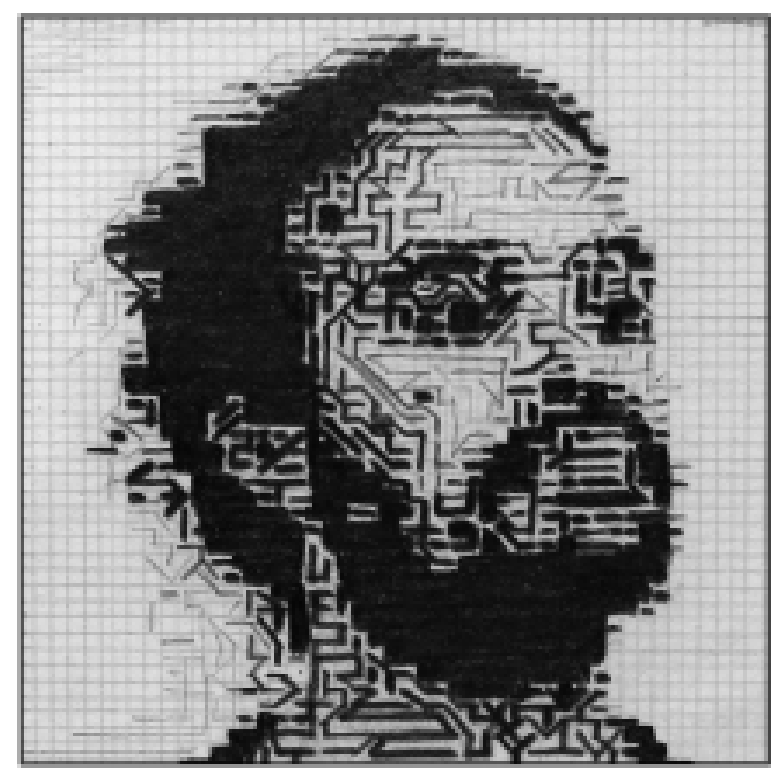

JB étude graphite, 2004, crayon sur papier, 8 x 8cm. 